\title{
A nomogram to predict the incidence of permanent stoma in elderly patients with rectal cancer
}

\author{
Chuangkun Li ${ }^{1,2 \#} \wedge$, Xiusen Qin ${ }^{1,2 \#} \wedge$, Zifeng Yang ${ }^{1,2 \#} \wedge$, Wentai Guo ${ }^{1,2} \wedge$, Rongkang Huang ${ }^{1,2}$, \\ Huaiming Wang ${ }^{1,2} \wedge$, Hui Wang ${ }^{1,2} \wedge$ \\ ${ }^{1}$ Department of Colorectal Surgery, The Sixth Affiliated Hospital, Sun Yat-sen University, Guangzhou, China; ${ }^{2}$ Guangdong Provincial Key \\ Laboratory of Colorectal and Pelvic Floor Disease, The Sixth Affiliated Hospital, Sun Yat-sen University, Guangzhou, China \\ Contributions: (I) Conception and design: Hui Wang, Huaiming Wang; (II) Provision of study materials of patients: X Qin, R Huang; (III) Collection \\ and assembly of data: C Li, Z Yang, W Guo; (IV) Data analysis and interpretation: C Li; (V) Administrative support: Hui Wang; (VI) Manuscript \\ writing: All authors; (VII) Final approval of manuscript: All authors. \\ \#These authors contributed equally to this work. \\ Correspondence to: Huaiming Wang; Hui Wang. Department of Colorectal Surgery, The Sixth Affiliated Hospital, Sun Yat-sen University, Guangzhou \\ 510655, China. Email: wanghm7@mail.sysu.edu.cn; wang89@mail.sysu.edu.cn.
}

\begin{abstract}
Background: Creation of a temporary diverting stoma during rectal cancer surgery is used widely to prevent undesirable outcomes related to anastomotic leakage (AL). The transition from temporary stoma (TS) to permanent stoma (PS) is a frequent outcome. Elderly patients may have a greater probability of PS. We aimed to identify risk factors of PS and developed a nomogram to predict the rate of PS for elderly patients. Methods: We enrolled elderly patients ( $\geq 70$ years) who underwent rectal cancer surgery with a TS between January 2014 and December 2017 at our hospital. We divided patients into two groups: a TS group and a PS group. We then identified the risk factors for PS and developed a nomogram to predict the possibility of PS. Results: Of the 278 elderly patients who received a diverting stoma, 220 (79.14\%) eventually underwent stoma reversal, and $58(20.86 \%)$ had PS. The proportion of males in the PS group was significantly higher than that of the TS group $(\mathrm{P}=0.048)$. Univariate and multivariate analysis showed that American Society of Anesthesiologists (ASA) score $(\mathrm{P}<0.001)$, laparotomy $(\mathrm{P}=0.004), \mathrm{AL}(\mathrm{P}<0.001)$, and tumor recurrence $(\mathrm{P}<0.001)$ were significantly correlated with PS. These four factors were included to construct the nomogram. The consistency index of the nomogram was 0.833 and the model yielded an area under the curve of 0.833 .
\end{abstract}

Conclusions: ASA score ( $\geq 3)$, laparotomy, AL, and tumor recurrence were independent risk factors for PS in elderly patients. Our nomogram exhibited moderate predictive ability.

Keywords: Rectal cancer; elderly patients; permanent stoma; nomogram

Submitted Dec 09, 2020. Accepted for publication Jan 19, 2021.

doi: $10.21037 /$ atm-21-29

View this article at: http://dx.doi.org/10.21037/atm-21-29

\footnotetext{
^ ORCID: Chuangkun Li, 0000-0002-1751-6324; Xiusen Qin, 0000-0001-8531-4858; Zifeng Yang, 0000-0001-9523-603X; Wentai Guo, 0000-0001-9305-3301; Rongkang Huang, 0000-0002-9499-0222; Huaiming Wang, 0000-0002-1976-3396; Hui Wang, 0000-0003-0086-5795.
} 


\section{Introduction}

Following rectal cancer surgery, between $1.6 \%$ and $20.5 \%$ of patients develop anastomotic leakage (AL) (1-4). Stoma was created to decrease the clinical level of AL. The choice between ileostomy and colostomy is controversial as patients who undergo ileostomy have a higher rate of stoma high flow output complications, while colostomy patients may have more stoma-related complications (1). However, ileostomy is more commonly performed clinically (5). Stoma reversal surgery is usually performed within 2-3 months after diverting stoma surgery (6). However, $6.1-27.5 \%(7-13)$ of patients fail to achieve stoma reversal, which eventually becomes a permanent stoma (PS). The continued existence of stoma may alter bowel habits, and could ultimately led to stoma-related complications including stoma hernia, stoma bleeding, stoma prolapse, dehydration, and electrolyte disorders $(14,15)$. Numerous studies $(4,8,16-18)$ investigating the risk factors for PS have found that comorbidities, surgical complications, and tumor recurrence were commonly associated with PS (17).

The rate of stoma closure in elderly rectal cancer patients is lower than that of younger patients, which generally occurs because the elderly population is more likely to suffer from stoma-related complications, comorbidities, poor general condition, and poor anorectal function (19). At present, there are only a few studies that focus on the reversal of diverting ileostomy for rectal cancer patients $(5,20-23)$. Thus, in this study, we evaluated the risk factors for developing PS in elderly patients aged 70 years and older and also this is the first time to report.

A nomogram is a statistical tool used for predicting clinical outcomes (24). Due to the practicality and convenience of its application, several nomograms have been developed to predict the prognosis in various cancers (24-26). Accordingly, a nomogram would help clinicians to predict the possibility of permanent diverting stoma for rectal cancer patients. Furthermore, prior studies only focused on predictors of PS after temporary stoma (TS) for patients of all ages $(4,6,8-10,17)$. In our study, we assessed the incidence of PS, identified independent risk factors, and constructed a predictive nomogram for elderly rectal cancer patients. The highlights of our study are that firstly, the research focus on the elderly patients, secondly, we pay attention to the situation of the un-reversal stoma after rectal cancer surgery which is related to the quality of life, and thirdly the clinician can find out the risk factors of permanent stoma and help solve clinical problems.

We present the following article in accordance with the TRIPOD reporting checklist (available at http://dx.doi. org/10.21037/atm-21-29).

\section{Methods}

\section{Patients}

We retrospectively collected the clinical data of elderly patients ( $\geq 70$ years old) with histologically confirmed rectal cancer who underwent tumor resection with diverting stoma between January 2014 and December 2018 at our hospital. The patients were divided into two groups according to the reversal of the stoma: a TS group and a PS group. The classification and pathological tumor node metastasis (TNM) staging were determined according to the American Joint Committee on Cancer (AJCC) guidelines (27). The timing of stoma reversal was determined by the clinical symptoms as well as findings from computed tomography (CT), magnetic resonance imaging (MRI), colonoscopy, and maximal squeeze pressure of the anus.

This study was conducted in accordance with the Declaration of Helsinki (as revised in 2013). Institutional review board statement: this study was reviewed and approved by the Ethics Committee of The Sixth Affiliated Hospital, Sun Yat-sen University (No. 2020ZSLYEC-078). Individual consent for this retrospective analysis was waived.

\section{Definition of stoma and follow-up}

The TS group comprised patients who received stoma reversal within 1 year after rectal cancer surgery. A PS was defined as a diverting stoma that failed to have a reversal procedure follow-up for at least 1 year $(8,12)$. Postoperative follow-up occurred every 3 months for 1 year. The main follow-up measures were as follows: general condition of the patient (mental condition, working condition, height, and body weight), stoma condition (stoma skin condition, stoma height, stoma-related complications), and anorectal function (daily frequency of defecation, shape of stool).

\section{Data comparison between TS and PS groups}

The following demographic data were collected and analyzed: age, sex, comorbidity, body mass index (BMI), American Society of Anesthesiologists (ASA) score, 


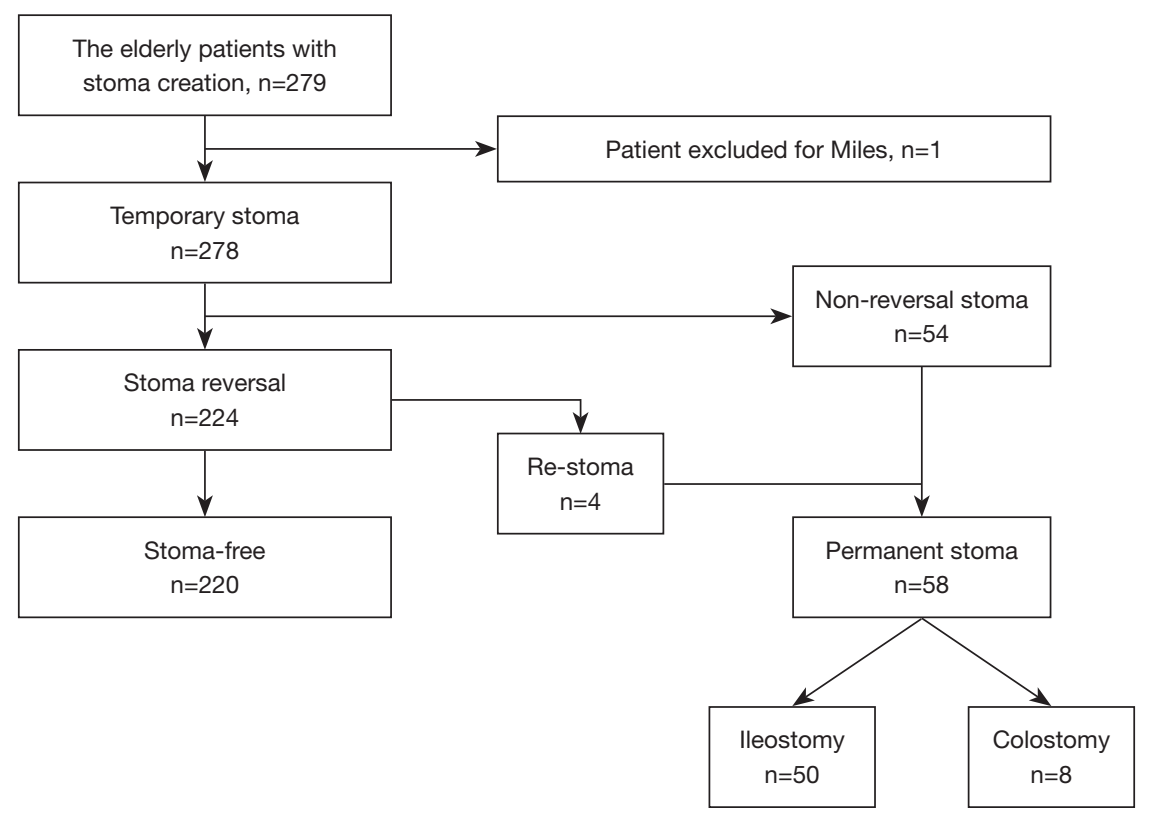

Figure 1 Flowchart detailing the selection of the patients in this study.

preoperative hemoglobin $(\mathrm{Hb})$, preoperative albumin (ALB), neoadjuvant therapy, surgical method (laparoscopy vs. open surgery), adjuvant therapy, metastasis, distance from anal margin, AL, type of stoma, anastomosis method (stapler $v s$. hand-sewn), and tumor recurrence.

\section{Statistical analysis}

Measurement data were expressed as the mean \pm standard deviation (SD) or median (minimum, maximum). MannWhitney rank sum tests or $t$-tests were used to compare the measurement data between the groups. IBM SPSS (version 25.0 for Windows; SPSS, Chicago, IL, USA) was used for logistic regressions to analyze the univariate and multivariate factors for PS. Variables with a $\mathrm{P}$ value $<0.1$ in the univariate analysis were selected for the multivariable model using logistic regression. The value $(<1)$ of the factors was the risk factor in the forest plots, which were constructed using GraphPad Prism (version 7 for Windows; GraphPad Software, CA, USA).

The nomogram was validated internally through 1,000 bootstrap resampling to calculate the estimated Harrell concordance index (C-index), thus indicating the performance of the model (27). In addition, we calibrated the nomogram using a calibration plot, which is a graphical representation of the relationship between the actual observed frequency and the predicted probability. The predictive performance of the nomogram was evaluated using the area under the receiver operating characteristic (ROC) curve (AUC) value. Differences were considered statistically significant when $\mathrm{P}<0.05$.

\section{Results}

\section{Patients}

As shown in Figure 1, 279 patients underwent rectal cancer surgery with diverting stoma between January 2014 and December 2018. One patient who underwent abdominoperineal resection was excluded from the study. Of the remaining patients, $220(79.14 \%)$ underwent stoma reversal, and $20.86 \%$ of the patients with diverting stoma failed to receive reversal surgery within 1 year after primary surgery. Ileostomy and colostomy were performed for $50(86.21 \%)$ and $8(13.79 \%)$ patients in the PS group, respectively. The mean follow-up time was 40 months.

The differences between the TS and PS groups are presented in Table 1. The TS group had a mean age of 73.79 years, and 147 (66.82\%) patients were male. The mean age in the PS group was 74.50 years, and 46 (79.31\%) patients were male. The proportion of males in the PS group was significantly higher than that of the TS group $(\mathrm{P}=0.048)$. Laparoscopic surgery was performed in 209 (95.00\%) patients in the TS group and $41(70.69 \%)$ patients in the PS 
Table 1 Patient characteristics between the TS and PS groups

\begin{tabular}{|c|c|c|c|}
\hline Characteristic & Temporary stoma & Permanent stoma & $P$ value \\
\hline Age (years) & $73.79 \pm 4.601$ & $74.50 \pm 5.020$ & 0.304 \\
\hline Sex & & & 0.048 \\
\hline Male & $147(66.82 \%)$ & $46(79.31 \%)$ & \\
\hline $\mathrm{BMI}\left(\mathrm{kg} / \mathrm{m}^{2}\right)$ & $22.25 \pm 2.97$ & $22.61 \pm 4.06$ & 0.523 \\
\hline Comorbidity & 75 (34.09\%) & $29(50 \%)$ & 0.021 \\
\hline ASA score & & & 0 \\
\hline 1 or 2 & 208 (94.55\%) & 37 (63.79\%) & \\
\hline Preoperative ALB (g/L) & $39.49 \pm 3.93$ & $38.88 \pm 3.69$ & 0.291 \\
\hline Neoadjuvant therapy & $60(27.27 \%)$ & $12(20.69 \%)$ & 0.456 \\
\hline Surgical method & & & 0 \\
\hline Laparoscopy & 209 (95.0\%) & $41(70.69 \%)$ & \\
\hline Open & $11(5.0 \%)$ & $17(29.31 \%)$ & \\
\hline Metastasis & & & 0.031 \\
\hline Yes & $18(8.18 \%)$ & $11(18.97 \%)$ & \\
\hline No & 202 (91.82\%) & 47 (81.03\%) & \\
\hline No & 212 (96.36\%) & $36(62.07 \%)$ & \\
\hline Type of stoma & & & 0.031 \\
\hline Ileum & 215 (97.73\%) & $50(86.21 \%)$ & \\
\hline Colon & $5(2.27 \%)$ & $8(13.79 \%)$ & \\
\hline Anastomosis method & & & 0.527 \\
\hline Stapler & $194(88.18 \%)$ & 49 (84.48\%) & \\
\hline Hand-sewn & $26(11.82 \%)$ & $9(15.52 \%)$ & \\
\hline Adjuvant therapy & $82(37.27 \%)$ & $17(29.31 \%)$ & 0.249 \\
\hline Tumor recurrence & $10(4.55 \%)$ & $12(20.69 \%)$ & 0.004 \\
\hline
\end{tabular}

$\mathrm{BMI}$, body mass index; $\mathrm{Hb}$, hemoglobin; ALB, preoperative albumin. 
Table 2 Univariate and multivariate logistic regression model representing risk factors for permanent stoma

\begin{tabular}{|c|c|c|c|}
\hline Variables & $\frac{\text { Univariate analysis }}{\mathrm{P}}$ & \multicolumn{2}{|c|}{ Multivariate analysis } \\
\hline Sex (male vs. female) & 0.069 & $1.719(0.696-4.247)$ & 0.240 \\
\hline Age (years) & 0.303 & - & - \\
\hline BMI $\left(\mathrm{kg} / \mathrm{m}^{2}\right)$ & 0.442 & - & - \\
\hline ASA score ( 1 or 2 vs. $\geq 3$ ) & 0.000 & $0.139(0.049-0.398)$ & 0.000 \\
\hline Preoperative $\mathrm{Hb}(\mathrm{g} / \mathrm{dL})$ & 0.378 & - & - \\
\hline Preoperative ALB (g/L) & 0.291 & - & - \\
\hline Neoadjuvant therapy (absent vs. present) & 0.455 & - & - \\
\hline Distance from anal margin $(\mathrm{cm})(>5 \mathrm{vs.} \leq 5)$ & 0.386 & - & - \\
\hline Anastomotic leakage (absent vs. present) & 0.000 & $0.069(0.024-0.197)$ & 0.000 \\
\hline Metastasis (absent vs. present) & 0.008 & $0.411(0.128-1.318)$ & 0.135 \\
\hline Adjuvant therapy (absent vs. present) & 0.261 & - & - \\
\hline Tumor recurrence (absent vs. present) & 0.000 & $0.139(0.047-0.417)$ & 0.000 \\
\hline
\end{tabular}

AUC, area under the curve; ROC, receiver operating characteristic; BMI, body mass index; Hb, hemoglobin; ALB, albumin; ASA, American Society of Anesthesiologists.

group $(\mathrm{P}<0.001)$. The percentage of $\mathrm{AL}$ was notably lower in the TS group ( $\mathrm{n}=8,3.64 \%)$ compared with the PS group $(\mathrm{n}=22,37.93 \%, \mathrm{P}<0.001)$. Furthermore, the preoperative ASA scores were markedly higher in the PS group $(\mathrm{P}<0.001)$. The rate of tumor recurrence and metastasis were also considerably higher in PS group (20.69\% vs. $4.55 \%$, $\mathrm{P}=0.004 ; 18.97 \%$ vs. $8.18 \%, \mathrm{P}=0.031$, respectively).

\section{Univariate and multivariate logistic regression analysis}

We assessed the risk factors for PS via univariate and multivariate analyses (Table 2). Univariate analysis showed that sex, comorbidity, surgical method, ASA score, AL, metastasis, and tumor recurrence were associated with PS. Multivariate analysis showed that ASA score ( $\geq 3$ ) [hazard ratio (HR): 0.139, 95\% confidence interval (CI): 0.049-0.398, $\mathrm{P}<0.001$ ], laparotomy (HR: 0.209, 95\% CI: 0.073-0.599, $\mathrm{P}=0.004)$, AL (HR: 0.069, 95\% CI: 0.024-0.197, $\mathrm{P}<0.001$ ), and tumor recurrence (HR: 0.139, 95\% CI: 0.047-0.417, $\mathrm{P}<0.001)$ were independent risk factors for PS. On the basis of these results, we developed a forest plot that was consistent with the results of the multivariate analysis (Figure 2).

\section{Nomogram development}

We developed a nomogram to predict the risk of PS using the four aforementioned independent factors (Figure 3). Using this nomogram, we could easily calculate the probability of PS. ROC analysis showed that the nomogram had considerable predictive potential. The AUC value of this model was 0.833 (Figure $4 A$ ), which was higher than that of any single factor (ASA score $=0.654$, tumor recurrence $=0.589$, surgical method $=0.622$, and $\mathrm{AL}$ $=0.671)$. We plotted the sum of each variable on the total point axis and obtained an estimated PS rate by drawing a vertical line from the drawn total point axis down to the result axis. The calibration curves between the predicted and actual observations internal validation demonstrated that the model was close to the ideal state, indicating good calibration (Figure 4B). The C-index value of this model was 0.833 , indicating moderate predictive ability for the risk of PS. 


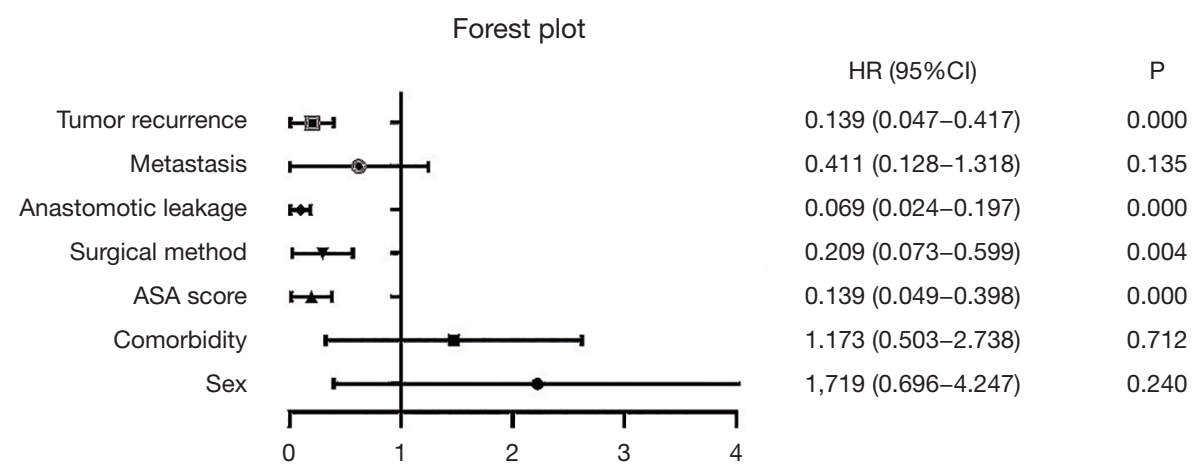

Figure 2 Forest plot.

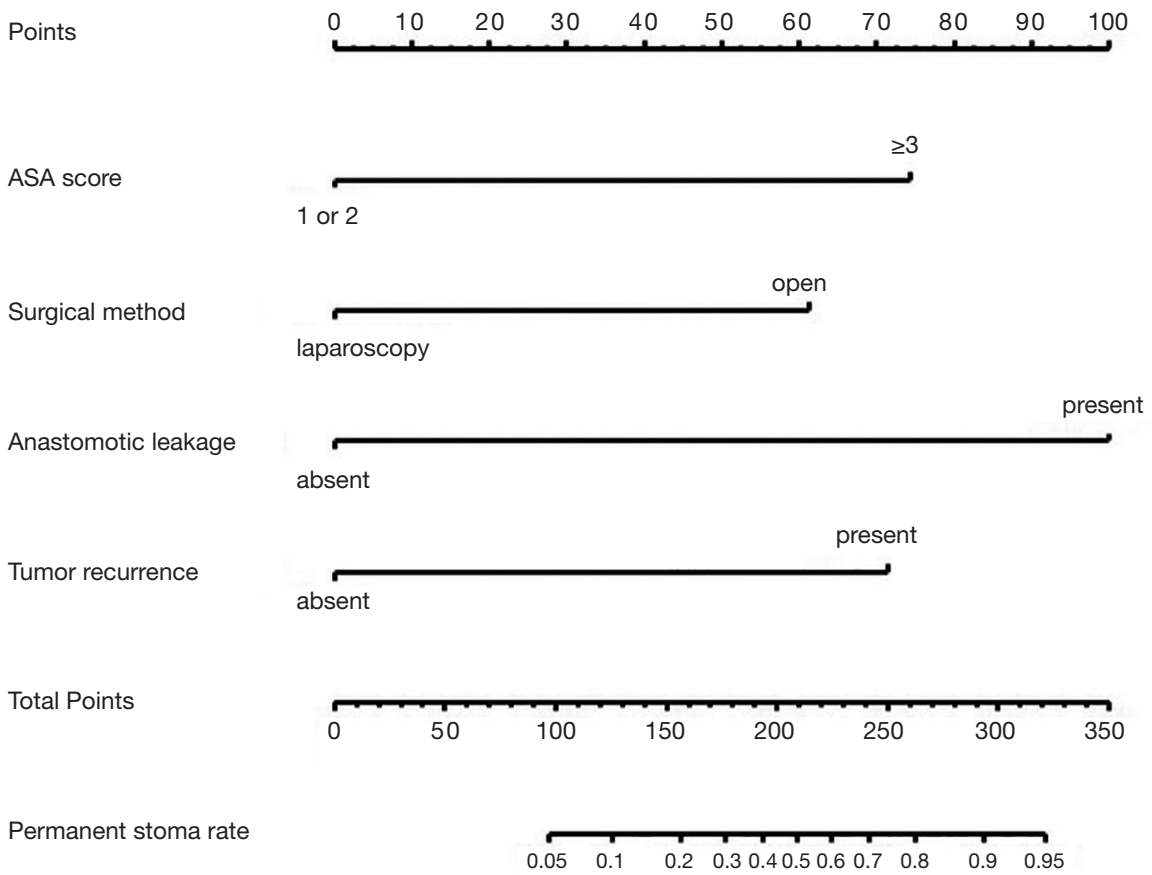

Figure 3 Nomogram for predicting permanent stoma (C-index: 0.833) after creation of a diverting stoma during rectal cancer surgery. The permanent stoma rate was estimated by adding the scores of anastomotic leakage, surgical method, ASA score, and tumor recurrence. ASA, American Society of Anesthesiologists.

\section{Discussion}

For patients with a high risk of AL following rectal cancer surgery, clinicians will consider performing diverting ileostomy or colostomy. However, there is currently no unified standard for selecting the appropriate procedure during rectal cancer surgery. Colostomy is usually employed during emergency surgeries, advanced tumor stages, and Hartman surgeries, which have high rates of mortality and morbidity (28), and results in a high incidence of PS (22). Overall, ileostomy is more commonly performed clinically (5). The reported incidence of irreversible stoma following rectal cancer surgery is $6.1-27.5 \%$ (7-13). Elderly patients who undergo rectal cancer surgery typically have a higher incidence of AL due to poor ASA scores (29) and comorbidities (30). Moreover, only a few studies have investigated the risk factors of PS in elderly patients following rectal cancer surgery. Therefore, we assessed 

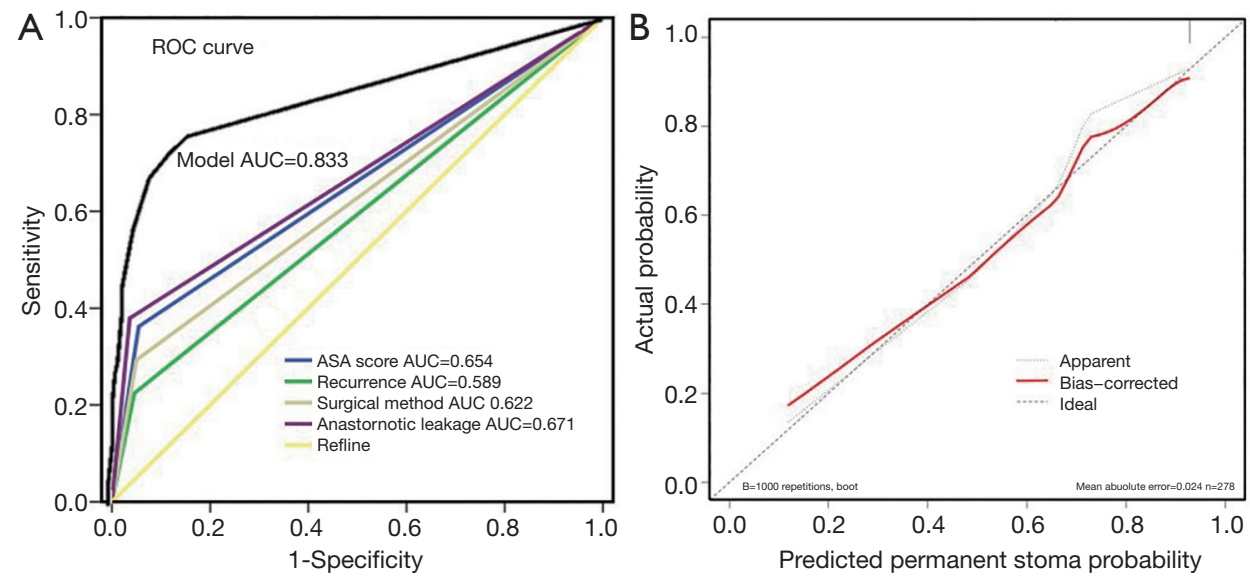

Figure 4 Using the independent factors to developed ROC curve and a calibration curve. (A) ROC curve for the nomogram; AUC was 0.833 (95\% CI =0.762-0.903). (B) Nomogram calibration curve. The $y$-axis represents the actual probability of PS. The $\mathrm{x}$-axis represents the predicted PS probability. The ideal line represents a perfect prediction model. The apparent line represents the performance of the nomogram, and a close fit to the ideal line represents a good prediction. AUC, area under the curve; ROC, receiver operating characteristic.

the incidence and risk factors of PS in elderly patients with rectal cancer. In this study, 58 (20.86\%) patients had PS during the follow-up period.

\section{Risk factors for permanent stoma}

Kim et al. (17) found that the incidence of PS was $25.0 \%$ and that distance from tumor to anal margin was the only risk factor for PS in patients with rectal cancer. In our study, PS developed in 58/278 (20.86\%) patients with rectal cancer, which included 54 patients in whom stoma was created during initial surgery and four patients who underwent re-stoma after stoma reversal. Postoperative AL was a common and severe complication in patients with rectal cancer, especially low and ultra-low rectal cancer, causing poor postoperative recovery, longer hospital stays, anorectal dysfunction, and even death (17). It has been reported that tumor recurrence and AL were common independent risk factors for PS (12). Zhou et al. (4) performed a meta-analysis of 10 studies with 8,568 rectal cancer patients. They found that rate of PS was $19 \%$, and that older age $(\mathrm{P}<0.001)$, ASA score $>2(\mathrm{P}<0.001)$, comorbidities $(\mathrm{P}<0.001)$, surgical complications $(\mathrm{P}<0.001)$, AL $(\mathrm{P}<0.001)$, stage IV tumor $(\mathrm{P}<0.001)$, and local tumor recurrence $(\mathrm{P}<0.001)$ were risk factors for PS. Mak et al. (18) focused on patients who underwent anterior resection for ultra-low rectal cancer and found that the 5-and 10 -year cumulative incidence of PS was $24.1 \%$ and $28.0 \%$, respectively. Patients with advanced cancer, adjuvant chemoradiotherapy, AL, and tumor recurrence were susceptible to PS. We focused on elderly patients, and found that AL, open surgery, ASA score $(\geq 3)$, and tumor recurrence were independent risk factors for PS. Thus, the most common causes of PS were AL and local tumor recurrence, which was consistent with previous study.

\section{Surgical method}

Zheng et al. (2) found that laparoscopic surgery reduced the incidence of AL compared with open surgery. Our study showed that the proportion of laparoscopic surgery was higher in the TS group compared with the PS group. In fact, the incidence of comorbidities was higher in elderly patients with rectal cancer, thus making it difficult to decide between laparoscopic surgery and open surgery for these patients (31). Compared with open surgery, laparoscopy surgery has the advantages of superior intraperitoneal field of vision and better protection for the abdominal wall. Furthermore, it has been reported that laparoscopic surgery is associated with a lower probability of stoma-related postoperative complications (1). Lewis et al. (32) studied the incidence of long-term stoma from a 6-year series of laparoscopic surgery in low rectal cancer and found that the rate of PS was lower than that of open surgery, which was consistent with the results of this study. We found that open surgery was a risk factor for PS, which could be 
explained by the fact that patients who had open surgery may have been more complicated, had previous surgeries, or had more distant tumors. Elderly patients had a higher frequency of comorbidities, because they were more likely to present with advanced tumors and complications, such as obstruction and perforation, which requires emergency surgery. There is a substantial body of evidence demonstrating that laparoscopic rectal cancer surgery could be performed safely in elderly patients without a significant increase in morbidity or mortality.

\section{ASA score and choice of stoma}

Compared to younger patients, elderly patients typically have a poorer ASA score. Although previous studies have assessed the risk factors for PS, only a few of these studies focused on elderly patients. The ASA score is a convenient method for assessing the degree of surgical risk and performance status of patients because it summarizes multiple patient characteristics, which are related to the patient's physical condition and age (31). Indeed, patients in our study with poor ASA scores had higher incidences of cardiovascular disease, diabetes, and other comorbidities. A recent study (33) showed that patients in a poor condition (ASA score $\geq 3$ ), elderly patients, and patients with advanced rectal cancer were more likely to develop PS, which is consistent with our findings.

Compared with other age cohorts, and due to the condition and particular comorbidities of elderly patients, diverting stoma in elderly patients are more likely to become PS. Moreover, patients who undergo ileostomy commonly have high-output complications postoperatively (6). A long history of ileostomy could also cause dehydration or renal insufficiency and affect quality of life, especially in elderly patients. Thus, we recommend that clinicians consider colostomy or Hartman surgery instead of ileostomy for elderly patients who have poor ASA scores.

\section{Nomogram}

Finally, a nomogram was developed to predict the risk of PS after rectal cancer surgery with diverting stoma for elderly patients using the four above-mentioned independent factors. At present, only a few predictive models of PS for elderly patients with rectal cancer have been published. The C-index of our nomogram was $0.833>0.7$, which indicated moderate predictive ability, and was higher than the result reported by Abe et al. $(\mathrm{C}$-index $=0.612)(23)$, whose study focused on the rate of stoma-reversal and was not aimed at elderly patients. The AUC of our model (0.833) was higher than that of any single factor (ASA score: 0.654; tumor recurrence: 0.589; surgical method: 0.622; and AL: 0.671), indicating that this model was more authentic. It can be used to assist with individualized therapy in the following ways. For elderly patients with a poor ASA score ( $\geq 3)$, open surgery and a high incidence of AL, and tumor recurrence after surgery, the rate of PS will be more than 0.9. For patients with a high rate of PS, a permanent colostomy can be considered as an alternative to with permanent ileostomy.

This study had several limitations that should be noted. Firstly, our study did not collect data regarding the quality of life of patients with or without PS. Secondly, we did not include the data of manometric parameters, which was important for evaluating anorectal function. Thirdly, due to a lack of definitive criteria for determining stoma creation and reversal, the preferences of clinicians and will of patients strongly affected the determination of stoma reversal. Furthermore, being a retrospective study, selection bias with regards to the type of procedure may present another limitation. Lastly, this was a single-center study with a small sample size, and thus, it would be useful to evaluate the effectiveness of our nomogram with more cases.

\section{Conclusions}

We found that open surgery, ASA score, AL, and tumor recurrence were independent risk factors for predicting PS in elderly patients following rectal cancer surgery. The nomogram developed in our study had moderate predictive ability and is a practical and promising tool that may help clinicians identify factors that affect the risk of PS in elderly patients following diverting stoma during rectal cancer surgery.

\section{Acknowledgments}

The authors thank AiMi Academic Services (www.aimieditor. com) and A. Kassem for English language editing and review services.

Funding: This work was supported by the Science and Technology Program of Guangzhou (201803010074).

\section{Footnote}

Reporting Checklist: The authors have completed the 
TRIPOD reporting checklist. Available at http://dx.doi. org/10.21037/atm-21-29

Data Sharing Statement: Available at http://dx.doi. org/10.21037/atm-21-29

Conflicts of Interest: All authors have completed the ICMJE uniform disclosure form (available at http://dx.doi. org/10.21037/atm-21-29). The authors have no conflicts of interest to declare.

Ethical Statement: The authors are accountable for all aspects of the work in ensuring that questions related to the accuracy or integrity of any part of the work are appropriately investigated and resolved. This study was conducted in accordance with the Declaration of Helsinki (as revised in 2013), and was approved by institutional ethics board of The Sixth Affiliated Hospital, Sun Yat-sen University (No. 2020ZSLYEC-078). Individual consent for this retrospective analysis was waived.

Open Access Statement: This is an Open Access article distributed in accordance with the Creative Commons Attribution-NonCommercial-NoDerivs 4.0 International License (CC BY-NC-ND 4.0), which permits the noncommercial replication and distribution of the article with the strict proviso that no changes or edits are made and the original work is properly cited (including links to both the formal publication through the relevant DOI and the license). See: https://creativecommons.org/licenses/by-nc-nd/4.0/.

\section{References}

1. Gavriilidis P, Azoulay D, Taflampas P. Loop transverse colostomy versus loop ileostomy for defunctioning of colorectal anastomosis: a systematic review, updated conventional meta-analysis, and cumulative meta-analysis. Surg Today 2019;49:108-17.

2. Zheng $\mathrm{H}, \mathrm{Wu} \mathrm{Z}, \mathrm{Wu} \mathrm{Y}$, et al. Laparoscopic surgery may decrease the risk of clinical anastomotic leakage and a nomogram to predict anastomotic leakage after anterior resection for rectal cancer. Int J Colorectal Dis 2019;34:319-28.

3. Kim CW, Baek SJ, Hur H, et al. Anastomotic Leakage After Low Anterior Resection for Rectal Cancer Is Different Between Minimally Invasive Surgery and Open Surgery. Ann Surg 2016;263:130-7.

4. Zhou X, Wang B, Li F, et al. Risk Factors Associated With
Nonclosure of Defunctioning Stomas After SphincterPreserving Low Anterior Resection of Rectal Cancer: A Meta-Analysis. Dis Colon Rectum 2017;60:544-54.

5. Rondelli F, Reboldi P, Rulli A, et al. Loop ileostomy versus loop colostomy for fecal diversion after colorectal or coloanal anastomosis: a meta-analysis. Int J Colorectal Dis 2009;24:479-88.

6. Yun JA, Cho YB, Park YA, et al. Clinical manifestations and risk factors of anastomotic leakage after low anterior resection for rectal cancer. Anz J Surg 2017;87:908-14.

7. Lee CM, Huh JW, Park YA, et al. Risk factors of permanent stomas in patients with rectal cancer after low anterior resection with temporary stomas. Yonsei Med J 2015;56:447-53.

8. Kim MJ, Kim YS, Park SC, et al. Risk factors for permanent stoma after rectal cancer surgery with temporary ileostomy. Surgery 2016;159:721-7.

9. Kim YA, Lee GJ, Park SW, et al. Multivariate Analysis of Risk Factors Associated With the Nonreversal Ileostomy Following Sphincter-Preserving Surgery for Rectal Cancer. Ann Coloproctol 2015;31:98-102.

10. Pan HD, Peng YF, Wang L, et al. Risk Factors for Nonclosure of a Temporary Defunctioning Ileostomy Following Anterior Resection of Rectal Cancer. Dis Colon Rectum 2016;59:94-100.

11. Celerier B, Denost Q, Van Geluwe B, et al. The risk of definitive stoma formation at 10 years after low and ultralow anterior resection for rectal cancer. Colorectal Dis 2016;18:59-66.

12. Lim SW, Kim HJ, Kim CH, et al. Risk factors for permanent stoma after low anterior resection for rectal cancer. Langenbecks Arch Surg 2013;398:259-64.

13. Kuryba AJ, Scott NA, Hill J, et al. Determinants of stoma reversal in rectal cancer patients who had an anterior resection between 2009 and 2012 in the English National Health Service. Colorectal Dis 2016;18:O199-205.

14. Wang FB, Pu YW, Zhong FY, et al. Laparoscopic permanent sigmoid stoma creation through the extraperitoneal route versus transperitoneal route. A metaanalysis of stoma-related complications. Saudi Med J 2015;36:159-63.

15. Herrle F, Sandra-Petrescu F, Weiss C, et al. Quality of Life and Timing of Stoma Closure in Patients With Rectal Cancer Undergoing Low Anterior Resection With Diverting Stoma: A Multicenter Longitudinal Observational Study. Dis Colon Rectum 2016;59:281-90.

16. Jutesten H, Draus J, Frey J, et al. High risk of permanent stoma after anastomotic leakage in anterior resection for 


\section{Page 10 of 10}

rectal cancer. Colorectal Dis 2019;21:174-82.

17. Kim S, Kim MH, Oh JH, et al. Predictors of permanent stoma creation in patients with mid or low rectal cancer: results of a multicentre cohort study with preoperative evaluation of anal function. Colorectal Dis 2020;22:399-407.

18. Mak JCK, Foo DCC, Wei R, et al. Sphincter-Preserving Surgery for Low Rectal Cancers: Incidence and Risk Factors for Permanent Stoma. World J Surg 2017;41:2912-22.

19. Koc U, Karaman K, Gomceli I, et al. Retrospective Analysis of Factors Affecting Early Stoma Complications. Ostomy Wound Manage 2017;63:28-32.

20. Klink CD, Lioupis K, Binnebosel M, et al. Diversion stoma after colorectal surgery: loop colostomy or ileostomy? Int J Colorectal Dis 2011;26:431-6.

21. Amelung FJ, Van T HC, Verheijen PM, et al. Ileostomy versus colostomy: which is preferable? Ned Tijdschr Geneeskd 2017;161:D788.

22. Klink CD, Willis S, Neumann UP, et al. Protective ileostoma versus protective transverse stoma. What evidence is available? Chirurg 2010;81:974-7.

23. Abe S, Kawai K, Nozawa H, et al. Use of a nomogram to predict the closure rate of diverting ileostomy after low anterior resection: A retrospective cohort study. Int J Surg 2017;47:83-8.

24. Kattan MW, Zelefsky MJ, Kupelian PA, et al. Pretreatment nomogram that predicts 5-year probability of metastasis following three-dimensional conformal radiation therapy for localized prostate cancer. J Clin Oncol 2003;21:4568-71.

25. Kawai K, Ishihara S, Yamaguchi H, et al. Nomograms for predicting the prognosis of stage IV colorectal cancer after curative resection: a multicenter retrospective study. Eur J Surg Oncol 2015;41:457-65.

Cite this article as: Li C, Qin X, Yang Z, Guo W, Huang R, Wang H, Wang H. A nomogram to predict the incidence of permanent stoma in elderly patients with rectal cancer. Ann Transl Med 2021;9(4):342. doi: 10.21037/atm-21-29

\section{Li et al. Nomogram to predict the incidence of permanent stoma}

26. Amin MB, Greene FL, Edge SB, et al. The Eighth Edition AJCC Cancer Staging Manual: Continuing to build a bridge from a population-based to a more "personalized" approach to cancer staging. CA Cancer J Clin 2017;67:93-9.

27. Harrell FE Jr, Califf RM, Pryor DB, et al. Evaluating the yield of medical tests. Jama 1982;247:2543-6.

28. Hackett NJ, De Oliveira GS, Jain UK, et al. ASA class is a reliable independent predictor of medical complications and mortality following surgery. Int J Surg 2015;18:184-90.

29. Sparreboom CL, van Groningen JT, Lingsma HF, et al. Different Risk Factors for Early and Late Colorectal Anastomotic Leakage in a Nationwide Audit. Dis Colon Rectum 2018;61:1258-66.

30. Benson AB, Venook AP, Al-Hawary MM, et al. Rectal Cancer, Version 2.2018, NCCN Clinical Practice Guidelines in Oncology. J Natl Compr Canc Netw 2018;16:874-901.

31. Park JH, Kim DH, Kim BR, et al. The American Society of Anesthesiologists score influences on postoperative complications and total hospital charges after laparoscopic colorectal cancer surgery. Medicine (Baltimore) 2018;97:e653.

32. Lewis OAO, McCallum IJD, Dixon S, et al. Longtermostomy as a quality marker: Comparison of outcomes from a six year series of laparoscopic surgery in MRI defined low rectal cancer. Int J Surg 2015;23:108-14.

33. Kim CH, Lee SY, Kim HR, et al. Nomogram Prediction of Anastomotic Leakage and Determination of an Effective Surgical Strategy for Reducing Anastomotic Leakage after Laparoscopic Rectal Cancer Surgery. Gastroenterol Res Pract 2017;2017:4510561.

(English Language Editor: A. Kassem) 